\title{
LA RELEVANCIA DE LA PREVISIBILIDAD JURÍDICA Algunas consideraciones a partir de Francisco Laporta y Liborio Hierro"
}

\author{
Isabel Lifante Vidal \\ Universidad de Alicante
}

\section{LA RELEVANCIA MORAL DEL IMPERIO DE LA LEY}

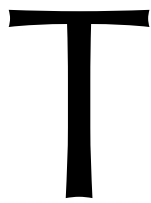

anto Francisco LAPORTA como Liborio HIERRO han argumentado en numerosas ocasiones sobre la relevancia moral del imperio de la ley, considerándolo un ideal ético-político al servicio de la autonomía personal. Francisco LAPORTA ha señalado que la existencia de normas que nos proporcionan un cierto grado de previsibilidad de las relaciones sociales es una condición necesaria (aunque no suficiente) ${ }^{1}$ para el desarrollo de la autonomía personal, entendida como la exigencia de que la vida del ser humano sea algo definido por él mismo en un marco de libertad personal y de racionalidad proyectiva. Y, en este sentido, afirma que «la fundamentación moral de todo el complejo mundo de normas e instituciones que constituyen lo que hoy designamos con el concepto de imperio de la ley no es otra que una apuesta moral implícita a favor de la autonomía personal» (LAPORTA, 2007: 18). Por su parte, Liborio HIERRO ha señalado, en un sentido muy similar, que la seguridad que un ordenamiento jurídico eficaz ofrece a sus destinatarios es el primer instrumento de la posibilidad de actuar libremente (HIERRO, 2003: 219).

Es difícil estar en desacuerdo con esta idea: la posibilidad de prever la conducta de otros sujetos, y en particular de los poderes públicos, es un requisito indispensable para poder desarrollar una vida autónoma. A partir de aquí, estos autores remarcan la importancia que, para la consecución de este ideal de previsibilidad, tienen lo que podemos considerar como rasgos formales del Derecho (el ideal de un Derecho compuesto por reglas generales y abstractas que puedan aplicarse a través de procedimientos subsuntivos). Las observaciones que a continuación realizo no pretenden ser una crítica a esta idea, sino una llamada de atención sobre la insuficiencia de estos rasgos formales para generar previsibilidad; no deben leerse por tanto como comentarios so-

* Este trabajo ha sido elaborado en el marco del proyecto de investigación «Desarrollo de una concepción argumentativa del Derecho» DER2013-42472-P, financiado por el Ministerio de Economía y Competitividad español.

1 En este sentido, LAPORTA, 2007: 52, recuerda que puede incluso haber normas cuyo contenido sea directamente contrario a cualquier promoción de la autonomía. 
bre sus tesis, sino «a partir» de ellas. No estoy muy segura de si Francisco LAPORTA o Liborio HIERRO estarán de acuerdo con mis observaciones, pero pese a ello no tengo ninguna duda de que las mismas — como gran parte de mi pensamiento iusfilosóficoson deudoras de sus enseñanzas.

\section{LA PREVISIBILIDAD: ¿DE QUÉ?, ¿PARA QUIÉN? $\mathrm{Y}_{¿ \text { HASTA CUÁNDO? }}$}

Con el término «previsibilidad» hacemos referencia a la cualidad de aquello cuyo acontecimiento puede ser conocido o conjeturado anticipadamente. Y es usual caracterizarla como una propiedad graduable, es decir, que puede presentarse en mayor o menor medida ${ }^{2}$. Ahora bien, cuando decimos que un Derecho (o, mejor dicho, una determinada regulación jurídica) puede proporcionarnos mayor o menor grado de previsibilidad, ¿qué es lo que estamos comparando? Para contestar a esta cuestión, debemos ser conscientes de una segunda característica de la previsibilidad: se trata de una propiedad que se proyecta en diversas dimensiones, cada una de las cuales resulta a su vez graduable. En particular, creo que deben destacarse al menos tres dimensiones $\left.^{3}: 1\right)$ una dimensión objetiva: ¿qué se puede prever?; 2) una dimensión subjetiva: ¿quién puede prever?, y 3) una dimensión temporal: ¿hasta cuándo se puede prever? Cada una de estas dimensiones justifica distintas exigencias: precisión, accesibilidad y estabilidad.

\subsection{La exigencia de precisión}

En la primera dimensión, una determinada normativa jurídica será tanto más previsible cuantas más cosas - o con mayor precisión - nos permita prever. De este modo, la gradualidad en esta primera dimensión dependerá de que se puedan conocer de antemano y con precisión tanto qué conductas son prohibidas, obligatorias o permitidas por el Derecho, como también las consecuencias jurídicas establecidas para ciertas conductas (o para ciertos estados de cosas), así como las condiciones para la generación de tales consecuencias (condiciones que pueden ser de índole procesal, temporal, económica, etc.).

Los análisis tanto de LAPORTA como de HIERRO parecen centrarse en esta primera dimensión, vinculando el grado de previsibilidad a la presencia de rasgos formales del Derecho: la formalidad en cuanto al origen, la formalidad en cuanto al contenido, la formalidad interpretativa y la formalidad perentoria ${ }^{4}$. Desde esta perspectiva, un De-

${ }^{2}$ Con ello no quiero decir que pueda adoptarse una métrica que nos permita cuantificar el grado de previsibilidad logrado, entre otras cosas porque en realidad cuando hablamos de previsibilidad no nos referimos exactamente a qué conductas o sus consecuencias hayan sido efectivamente previstas, sino que hayan podido serlo, es decir, que se den las condiciones para posibilitar dicho conocimiento anticipado.

3 Podríamos añadir una cuarta dimensión: la fiabilidad de las previsiones. Vinculada a esta dimensión, aparecería la exigencia de eficacia del Derecho, sobre la que Liborio HIERRO ha llamado la atención con especial énfasis (HIERRO, 2003).

${ }^{4}$ Cfr. AtiYah y Summers, 1987: 11 y ss. 
recho será tanto más previsible cuanto la aplicación de sus normas — que han de ser identificadas por su origen autoritativo- pueda realizarse de manera independiente de las razones sustantivas que las justifican (para lo que han de adoptar la forma de reglas de acción y gozar de una completa autonomía semántica), y su interpretación se limite a constatar su significado literal. La situación óptima parecería ser entonces aquella en la que, para la aplicación del Derecho, bastara con llevar a cabo un mero razonamiento deductivo ${ }^{5}$. Cualquier término valorativo, o cualquier atribución de un poder discrecional, o incluso cualquier actividad interpretativa menoscabaría la previsibilidad así entendida.

Pero hemos de ser conscientes de que hay ocasiones en las que la aplicación del Derecho solo exija un razonamiento deductivo a partir de premisas normativas preexistentes tampoco nos asegura poder prever las consecuencias de nuestra conducta. Pensemos en la concesión de ayudas a través de un baremo muy formalizado, pero en el que la cuantía a repartir sea fija, de modo que la ayuda a recibir por cada sujeto dependerá del número total de solicitantes (dato imprevisible para los solicitantes). Y, por otro lado, la exigencia de que todas las decisiones sean predecibles a través de procedimientos meramente subsuntivos, además de ser imposible de conseguir, ni siquiera parece ser un objetivo deseable. En muchos casos parece conveniente optar por cierta flexibilidad en la regulación jurídica (lo que implicaría un bajo grado de formalidad en cuanto al contenido). Pensemos en los supuestos en los que lo que resulta jurídicamente relevante prever es que un determinado interés (p. ej., el bienestar de un menor) resultará protegido y no tanto cómo lo será. En estos casos, el Derecho puede regular la conducta a través de la delegación de poderes discrecionales, para que sean los aplicadores los que - a la luz de las circunstancias de cada caso- adopten las medidas más adecuadas. Para ello habrá que llevar a cabo razonamientos de adecuación medio a fin y razonamientos ponderativos, que van más allá de la subsunción, pero que no están exentos de posibilidad de control racional y, en ese sentido, no excluyen cierto grado de previsibilidad.

\subsection{La exigencia de accesibilidad}

La segunda dimensión, la subjetiva, hace referencia a la «extensión» de la previsibilidad: qué sujetos están en situación de poder llevar a cabo las previsiones. Una normativa jurídica generará mayor grado de previsibilidad en este sentido cuanto más fácilmente los ciudadanos puedan realizar dichas previsiones a partir de ella. El primer requisito que aparece vinculado a esta dimensión es la exigencia de publicidad de las normas y su accesibilidad. Una norma secreta o de acceso restringido implicaría la negación absoluta de la previsibilidad en esta dimensión. Pero, por supuesto, la ac-

5 LAPORTA considera que el objeto de la previsibilidad que interesa al Derecho no sería tanto hechos que van a ocurrir, como «corolarios que pueden ser inferidos y comprobados» (el contenido proposicional de la decisión judicial). A partir de aquí, señala que cuanto más seguro sea el razonamiento que dé lugar a la conclusión en que ha de consistir el contenido proposicional de la decisión judicial, más predecible será la misma, y esto ocurre —en su opinión - cuando nos encontramos ante razonamientos deductivos, pues si necesitan de otro tipo de razonamientos (evaluativos y/o ponderativos) el paso de las premisas a la conclusión ya no sería necesario (LAPORTA, 2007: 38 y ss.). 
cesibilidad también es graduable, dependiendo de una serie de factores muy diversos que pueden facilitarla o dificultarla, pensemos en la publicación en medios de pago, o la publicación en tablones de anuncios ubicados en dependencias con horarios reducidos, o la publicación de convocatorias universitarias en periodo vacacional, etc. Pero junto a este aspecto «formal» de la accesibilidad de las normas nos encontramos con otra dimensión más sustantiva que es la que se refiere a que los ciudadanos puedan «efectivamente» conocer y comprender adecuadamente el Derecho aplicable. Los factores que aquí pueden influir son muy diversos. Pensemos en la claridad de la redacción, el nivel de complejidad técnica, las remisiones legislativas, la dispersión de competencias normativas (que implican la necesidad de conocer previamente qué administración pública es competente para una determinada materia), etc. Todos estos factores pueden llegar a hacer muy complejo -incluso prácticamente imposibilitaral ciudadano lego (incluso al jurista no muy especializado) conocer o comprender al detalle la regulación aplicable.

Estas mismas razones que avalan la imposibilidad de conocer al detalle todo el Derecho, son las que hacen que revista especial importancia un factor generador de seguridad jurídica: la coherencia normativa que nos ofrecen los principios jurídicos. Si distinguimos entre la seguridad de los legos y la de los abogados, habría que admitir que la previsibilidad jurídica para el lego no depende tanto de una predeterminación total del contenido de las decisiones judiciales en reglas jurídicas preexistentes, sino que lo que permite orientarse a la población en general, y lo que por tanto contribuye a generar la previsibilidad necesaria para que puedan desarrollar satisfactoriamente sus planes de vida, es sobre todo el reconocimiento de los principios que subyacen a las reglas; y, en último término, la congruencia entre las normas jurídicas y los modos de vida o la proximidad entre las normas jurídicas y las normas sociales ${ }^{6}$.

Si somos conscientes de esta relevancia de los principios, entenderemos que también las razones sustantivas, y no solo las formales, cumplen un papel fundamental en la generación de previsibilidad. Serían estas mismas razones las que nos llevan, por ejemplo, a aceptar los argumentos teleológicos y valorativos en la interpretación jurídica, justificando el desplazamiento de uno de los tipos de formalidad (la interpretativa); o las figuras de fraude de ley, abuso de derecho y desviación de poder (los denominados ilícitos «atípicos»), y que supondrían el desplazamiento de otro tipo de formalidad: la perentoria.

Hasta ahora hemos hablado de que una determinada regulación jurídica sería tanto más previsible cuantos más sujetos puedan realizar previsiones a partir de ella; pero en realidad lo importante no es el «número» de sujetos en abstracto, sino en relación con los sujetos potencialmente afectados por la regulación. Y en este sentido hay que tener en cuenta que hay regulaciones que afectan típicamente a «clases» de sujetos con intereses enfrentados y con distintas posibilidades de «acceso real» al conocimiento de la normativa: ciudadanos/administración, consumidores/proveedores. En ocasiones una misma normativa puede generar grados distintos de previsibilidad para cada una de las clases de sujetos afectados. Pensemos, por ejemplo, en el Derecho de los

\footnotetext{
${ }^{6}$ Cfr. Llewellyn, 1933: 58.
} 
consumidores, que parece estar diseñado precisamente a partir de esta contraposición entre clases de sujetos que presentan una diferente accesibilidad tanto a la regulación, como a la información relevante para la determinación de las consecuencias de dicha regulación.

\subsection{La exigencia de estabilidad}

La tercera dimensión de la previsibilidad sería la temporal. Desde esta perspectiva una determinada normativa generará mayor previsibilidad cuanto mayor sea el lapso de tiempo para el que nos permite realizar previsiones. La retroactividad supondría, obviamente, la negación de la previsibilidad en esta dimensión; pero aquí se justifica también la exigencia de estabilidad normativa.

Ahora bien, podemos distinguir dos sentidos de estabilidad, conectados ambos con la previsibilidad. El primer sentido, más formal, entiende la estabilidad como ausencia de cambios: las normas jurídicas han de tener un mínimo de perdurabilidad en el tiempo, para que los destinatarios puedan planificar su conducta a medio o largo plazo. Desde esta perspectiva las transformaciones jurídicas frecuentes generan déficit de previsibilidad. Para el segundo sentido (ya no tan formal), la estabilidad se entiende como continuidad (coherencia), y no como mera ausencia de cambios. En este segundo sentido tendremos que entrar a valorar el contenido de los cambios realizados para determinar si los mismos implican o no inestabilidad y, por tanto, si afectan o no a la seguridad jurídica. Una determinada regulación puede ser más o menos estable en el primero y/o en el segundo de los sentidos. En ocasiones, ciertos cambios normativos (es decir, cierto déficit de estabilidad en el primer sentido) pueden no solo no afectar a la previsibilidad, sino incluso incrementarla. Supongamos que una regla tiene una formulación ambigua y que la reforma elimina dicha ambigüedad; o que la reforma consiste en eliminar una regla que resultaba incoherente con otras reglas o con algunos principios jurídicos.

Para dar cuenta de esta dimensión de la previsibilidad no basta con la incorporación de una perspectiva diacrónica del Derecho, según el cual se trataría de un sistema dinámico, es decir, un conjunto de elementos que cambia en el tiempo; sino que es imprescindible considerarlo como una práctica social que se desarrolla a lo largo del tiempo y que está dirigida a la persecución de ciertos objetivos considerados valiosos. Desde esta perspectiva, la práctica ha de presentar una cierta continuidad, al menos en sus principios fundamentales. Pues son precisamente los valores que pretende desarrollar la práctica los que nos permiten identificarla como un todo coherente dotado de sentido (es decir, dirigido a la persecución de ciertos fines).

\section{3. ¿ES LA PREVISIBILIDAD DEPENDIENTE SOLO DE RASGOS FORMALES DEL DERECHO?}

Como acabamos de ver, una determinada regulación puede resultar muy precisa (tener un alto grado de previsibilidad en la primera dimensión), pero sin embargo 
ser muy poco accesible para sus destinatarios, o muy variable como para permitir hacer previsiones a largo plazo; o por el contrario, una regulación no tan precisa puede resultar más accesible y estable, etc. Lo que en ocasiones incrementa el nivel de previsibilidad en una de las dimensiones puede también incrementarla en las otras, pero también pueden existir relaciones de proporcionalidad inversa (a mayor precisión, menor accesibilidad, o menor estabilidad). Pero además estas dimensiones son a su vez complejas, y lo que - por ejemplo- genera previsibilidad para una clase de sujetos (digamos los proveedores), puede destruirla para otra clase de sujetos (los consumidores); o una formulación muy formal en cuanto al contenido puede generar incoherencias con otras normas que hagan necesario en alguna ocasión apartarse de la interpretación literal.

Por tanto, la cuestión crucial no será determinar si se ve afectada o no la previsibilidad en abstracto, sino cuál es la previsibilidad que consideramos jurídicamente valiosa. Necesitamos establecer cuáles son las expectativas que merecen ser jurídicamente protegidas; en este sentido podemos redefinir la seguridad jurídica en términos de protección de expectativas razonablemente fundadas de los ciudadanos (es decir, expectativas que han de ser consideradas legítimas a la luz de los principios y valores del propio Derecho). La previsibilidad que consideramos valiosa y la que por tanto el principio de seguridad jurídica nos obliga a maximizar sería entonces aquella que afecta a expectativas jurídicas razonablemente fundadas (es decir, al menos prima facie legítimas para el propio ordenamiento a la luz de los principios y valores reconocidos por el propio Derecho). Si esto es así, la previsibilidad jurídica no puede seguir siendo concebida como vinculada únicamente a los rasgos formales del Derecho, al margen de su contenido.

\section{BIBLIOGRAFÍA}

ATIENZA, M., 1985: Introducción al Derecho, Barcelona: Barcanova.

— 2013: «Seguridad jurídica y formación judicial», ponencia presentada en el I Congreso bienal. Seguridad jurídica y democracia en Latinoamérica, Girona, 3-5 de junio de 2013.

- 2015: «Entrevista a Francisco Laporta», en Doxa. Cuadernos de Filosofía del Derecho, 38, 557-577.

Atiyah, P. S., y Summers, R., 1987: Form and Substance in Anglo-American Law, Oxford: Clarendon Press.

GOMETZ, G., 2012: La certeza jurídica como previsibilidad, trad. D. MORENO CRUZ y D. DEI VECCHI, Madrid: Marcial Pons.

HiERro, L., 2003: La eficacia de las normas jurídicas, Barcelona: Ariel.

- 2016: «Seguridad jurídica, crisis de la ley y crisis de la aplicación de la ley», ponencia presentada en el I Congreso de filosofía del Derecho para el mundo latino, Alicante, mayo de 2016.

LAPORTA, F. J., 1994: «El imperio de la ley», en Doxa. Cuadernos de Filosofía del Derecho, 15-16, 1994, 133-145.

- 2007: El imperio de la ley. Una visión actual, Madrid: Trotta.

LIFANTE VIDAL, I., 2013: «Seguridad jurídica y previsibilidad», en Doxa. Cuadernos de filosofía del Derecho, 36, 85-105. 
Llewellyn, K. N., 1933: Präjudizienrecht und Rechtsprechung in Amerika, Eine Spruchauswabl mit Besprechung, Leipzig: Weicher.

Summers, R., 1993: «A Formal Theory of the Rule of Law», en Ratio Iuris, vol. 6, 2 de julio de 1993, 127-142.

- 2006: Form and Function in a Legal System. A General Study, New York: Cambridge University Press. 